\title{
Lower bounds on the growth of Sobolev norms in some linear time dependent Schrödinger equations
}

\author{
A. Maspero*
}

October 10, 2018

\begin{abstract}
In this paper we consider linear, time dependent Schrödinger equations of the form i $\partial_{t} \psi=K_{0} \psi+V(t) \psi$, where $K_{0}$ is a positive self-adjoint operator with discrete spectrum and whose spectral gaps are asymptotically constant.

We give a strategy to construct bounded perturbations $V(t)$ such that the Hamiltonian $K_{0}+V(t)$ generates unbounded orbits. We apply our abstract construction to three cases: (i) the Harmonic oscillator on $\mathbb{R}$, (ii) the half-wave equation on $\mathbb{T}$ and (iii) the Dirac-Schrödinger equation on Zoll manifolds. In each case, $V(t)$ is a smooth and periodic in time pseudodifferential operator and the Schrödinger equation has solutions fulfilling $\|\psi(t)\|_{r} \gtrsim|t|^{r}$ as $|t| \gg 1$.
\end{abstract}

\section{Introduction}

In this paper we study linear Schrödinger equations of the form

$$
\mathrm{i} \partial_{t} \psi=K_{0} \psi+V(t) \psi
$$

on a scale of Hilbert spaces $\mathcal{H}^{r}$. Here $K_{0}$ is a positive, selfadjoint operator with purely discrete spectrum, $V(t)$ is a time dependent self-adjoint perturbation, and the scale $\mathcal{H}^{r}$ is the one defined spectrally by $K_{0}$.

We develop an abstract technique to construct smooth and periodic in time, bounded operators $V(t)$ for which (1) has unbounded orbits with norms growing polynomially in time, despite every orbit of the unperturbed flow being bounded.

\footnotetext{
*International School for Advanced Studies (SISSA), Via Bonomea 265, 34136, Trieste, Italy Email: alberto.maspero@sissa.it
} 
Our main result (Theorem 2.5) is to develop a general procedure to construct such perturbations in case $K_{0}$ has constant spectral gaps.

We successfully apply this strategy to three different models: (i) the Harmonic oscillator on $\mathbb{R}$, (ii) the half-wave equation on $\mathbb{T}$ and (iii) the Dirac-Schrödinger equation on Zoll manifolds. In each case, we construct $V(t)$ as a pseudodifferential operator of order 0 , smooth and $2 \pi$-periodic in time, so that the Hamiltonian $K_{0}+V(t)$ has solutions $\psi(t)$ fulfilling

$$
\|\psi(t)\|_{r} \geq C_{r} t^{r}, \quad \text { for } t \gg 1
$$

which display, therefore, growth of Sobolev norms.

Note that case (iii) differs from (i) and (ii), since the Dirac-Schrödinger operator on Zoll manifolds has only asymptotically constant spectral gaps; however, such operator is a smoothing perturbation of a $K_{0}$ with constant spectral gaps, and our method applies with just a minor modification. In particular, the difference between cases (i)-(ii) and (iii) is that in the former ones the perturbations $V(t)$ can be arbitrary small in size; on the contrary, in case (iii) $V(t)$ has to contain a not perturbative term to correct the spectral gaps.

The problem of constructing unbounded solutions in Schrödinger equations has recently attracted a lot of attention. However, even in the simpler case of linear time dependent equations there are not many results in the literature. Up to our knowledge, the only examples were given by Bourgain for a Klein-Gordon and Schrödinger equation on $\mathbb{T}$ Bou99, by Delort for the Harmonic oscillator on $\mathbb{R}$ [Del14, and by Bambusi, Grébert, Robert and the author for the Harmonic oscillators on $\mathbb{R}^{d}, d \geq 1$ [BGMR18. While the techniques of [Bou99, Del14] are quite involved, the construction of [BGMR18] is simpler and based on a result by Graffi and Yajima GY00 to prove stability of the absolutely continuous spectrum of a certain Floquet operator.

It turns out that the mechanism of Graffi and Yajima is quite general, and the main idea of this paper is that their procedure can be "reversed", in an abstract setting, to create perturbations which provoke growth of norms. It is therefore worth to spend few words on the result by Graffi and Yajima. GY00] considers the following periodically forced Harmonic oscillator

$$
\mathrm{i} \dot{\psi}=\frac{1}{2}\left(-\partial_{x x}+x^{2}\right) \psi+a x(\sin t) \psi, \quad x \in \mathbb{R}, a \in \mathbb{R} \backslash\{0\},
$$

as an example of Hamiltonian whose Floquet spectrum is absolutely continuous, despite the fact that the unperturbed Hamiltonian has discrete spectrum. This statement is proved by conjugating (3) by the flow of the unperturbed Hamiltonian and then by a 
Galilean transform; the conjugated Hamiltonian is simply $\frac{a}{2 \mathrm{i}} \partial_{x}$, whose Floquet spectrum is absolutely continuous. In [BGMR18, this scheme is exploited to prove growth of norms for (3), by simply remarking that $\frac{a}{2 \mathrm{i}} \partial_{x}$ generates orbits unbounded w.r.t. the norms defined spectrally by the harmonic oscillator (actually [BGMR18] considers also perturbations quasi-periodic in time).

Now, the main idea of this paper is that it is possible to "reverse" the mechanism of GY00 to construct time dependent equations with unbounded orbits. The construction is the following: assume to have a time independent pseudodifferential operator $A$ whose propagator generates unbounded orbits, and define the time dependent perturbation

$$
V_{A}(t):=e^{-\mathrm{i} t K_{0}} A e^{\mathrm{i} t K_{0}}
$$

(i.e. conjugate $A$ by the inverse flow of the unperturbed operator). By construction, the dynamics of $K_{0}+V_{A}(t)$ is unitarily equivalent to the dynamics of $A$, so it has unbounded orbits as well. Provided the map $t \mapsto V_{A}(t)$ is smooth and periodic in time, we have found our perturbation; it is here that we use (indirectly) that $K_{0}$ has constant spectral gaps, see Remark 2.4.

Therefore, all is left to do in applications is to find a time independent operator whose propagator generates unbounded orbits. This is a much simpler task, and the general philosophy is to look for operators with nontrivial absolutely continuous spectrum; indeed, for these operators, the Guarneri-Combes theorem [Gua89, Com93] guarantees the existence of initial data $\psi$ for which the time-averaged Sobolev norms $\frac{1}{T} \int_{0}^{T}\left\|e^{-\mathrm{i} t A} \psi\right\|_{r} \mathrm{~d} t$ grow in $T$. This is a slightly weaker statement than (2); however, in applications, one can typically prove the stronger estimate $\left\|e^{-i t A} \psi\right\|_{r} \geq C_{r, \psi} t^{r}$ as $t \rightarrow \infty$. In particular we succeed in doing this for the Harmonic oscillator on $\mathbb{R}$, giving an alternative, shorter proof of Delort's result [Del14] (see Theorem 3.4).

As a further comment, it is interesting to compare the rate of growth (21) with the upper bounds proved in MR17, BGMR17 for equations of the form (1). In particular, the results of [MR17] imply that for any $V(t)$ continuous in time (but otherwise arbitrary depending) and pseudodifferential of order $\rho \leq 1$, each solution of (1) fulfills

$$
\|\psi(t)\|_{r} \leq C_{r, \rho}\langle t\rangle^{\frac{r}{1-\rho}}, \quad \forall t \in \mathbb{R} .
$$

Therefore, the solutions we construct here saturate (5), at least for $\rho=0$.

The upper bound (51) can be improved adding the assumption that $V(t) \equiv V(\omega t)$ is 
quasiperiodic in time with a nonresonant frequency vector $\omega \in \mathbb{R}^{n}$. Indeed, in BGMR17] it is proved that if $\omega$ fulfills the nonresonance condition

$$
\exists \gamma, \tau>0 \text { s.t. }|\ell+\omega \cdot k| \geq \frac{\gamma}{(1+|k|+|\ell|)^{\tau}}, \quad \forall \ell \in \mathbb{Z} \backslash\{0\}, \forall k \in \mathbb{Z}^{n} \backslash\{0\},
$$

then for any $r \geq 0$, any $\epsilon>0$ arbitrary small, (15) improves to

$$
\|\psi(t)\|_{r} \leq C_{r, \epsilon}\langle t\rangle^{\epsilon}, \quad \forall t \in \mathbb{R}
$$

the last estimate means that the growth of norms, if happens, is subpolynomial in time. Note that (7) is not in contrast with the faster growth of the norms (2) ; indeed the spectral condition that we impose on $K_{0}$ (see (9)) implies that $V_{A}(t)$ defined in (4) is periodic in time with frequency $\omega=1$, which is clearly resonant.

Finally, in some cases one can prove that the Sobolev norms of the solution stay uniformly bounded in time. This requires typically nonresonance conditions stronger than (6) and a smallness assumption on the size of the perturbation. In this case, one might try to prove a reducibility KAM theorem, conjugating $K_{0}+V(t)$ to a new Hamiltonian which is time independent and commutes with $K_{0}$; as a consequence, one gets the upper bound

$$
\sup _{t \in \mathbb{R}}\|\psi(t)\|_{r} \leq C_{r}
$$

Concerning the systems that we treat here, the reducibility scheme has been successfully implemented for the Harmonic oscillators on $\mathbb{R}^{d}$ Com87, Wan08, GT11, GP16b, Bam18, BGMR18, wave equations on the torus [CY00, FHW14, Mon17] (these methods can be used to prove reducibility for the half-wave equation on $\mathbb{T}$ ), and Klein-Gordon equation on the sphere GP16a. In all cases the frequency $\omega$ must be chosen in a Cantor set of nonresonant vectors and the perturbation must be sufficiently small in size. We recall that also the perturbations constructed here (and which provoke growth of norms) can be arbitrary small in size; therefore the stability/instability of the system depends only on the resonance property of the frequency $\omega$.

Before closing this introduction, let us mention that, in case of nonlinear Schrödinger equations, the problem of constructing solutions with unbounded orbits is extremely difficult. A first breakthrough was achieved in [CKSTT], which constructs solutions of the cubic nonlinear Schrödinger equation on $\mathbb{T}^{2}$ whose Sobolev norms become arbitrary large (see also [Han14, Gua14, HP15, GHP16] for generalizations of this result); however the existence of unbounded orbits for this model is still an open problem.

At the moment, existence of unbounded orbits has only been proved by Gérard and 
Grellier [GG17] for the cubic Szegő equation on $\mathbb{T}$, and by Hani, Pausader, Tzvetkov and Visciglia [HPTV15] for the cubic NLS on $\mathbb{R} \times \mathbb{T}^{2}$.

Acknowledgments. We wish to thank Dario Bambusi, Didier Robert and Benoît Grébert for many useful discussions and suggestions during the preparation of the manuscript. Currently we are partially supported by PRIN 2015 "Variational methods, with applications to problems in mathematical physics and geometry".

\section{An abstract theorem of growth}

We start with a Hilbert space $(\mathcal{H},\langle\cdot, \cdot\rangle)$ and a reference operator $K_{0}$, which we assume to be selfadjoint and positive, namely such that

$$
\left\langle\psi ; K_{0} \psi\right\rangle \geq c_{K_{0}}\|\psi\|_{0}^{2}, \quad \forall \psi \in \operatorname{Dom}\left(K_{0}^{1 / 2}\right), \quad c_{K_{0}}>0
$$

and define as usual a scale of Hilbert spaces by $\mathcal{H}^{r}=\operatorname{Dom}\left(K_{0}^{r}\right)$ (the domain of the operator $K_{0}^{r}$ ) if $r \geq 0$, and $\mathcal{H}^{r}=\left(\mathcal{H}^{-r}\right)^{\prime}$ (the dual space) if $r<0$. We endow $\mathcal{H}^{r}$ with the norm

$$
\|\psi\|_{r}:=\left\|K_{0}^{r} \psi\right\|_{0}
$$

where $\|\cdot\|_{0}$ is the norm of $\mathcal{H}^{0} \equiv \mathcal{H}$. Finally we denote by $\mathcal{H}^{-\infty}=\bigcup_{r \in \mathbb{R}} \mathcal{H}^{r}$ and $\mathcal{H}^{+\infty}=\bigcap_{r \in \mathbb{R}} \mathcal{H}^{r}$. Notice that $\mathcal{H}^{+\infty}$ is a dense linear subspace of $\mathcal{H}^{r}$ for any $r \in \mathbb{R}$ (this is a consequence of the spectral decomposition of $K_{0}$ ).

Remark 2.1. By the very definition of $\mathcal{H}^{r}$, the unperturbed flow $e^{-\mathrm{i} t K_{0}}$ preserves each norm, $\left\|e^{-i t K_{0}} \psi\right\|_{r}=\|\psi\|_{r} \forall t \in \mathbb{R}$. Consequently, every orbit of the unperturbed equation is bounded.

For $\mathcal{X}, \mathcal{Y}$ Banach spaces, we denote by $\mathcal{L}(\mathcal{X}, \mathcal{Y})$ the set of bounded operators from $\mathcal{X}$ to $\mathcal{Y}$; if $\mathcal{X}=\mathcal{Y}$, we simply write $\mathcal{L}(\mathcal{X})$.

We state now the assumptions that we need in order to prove our result. The first one is a spectral property of $K_{0}$ :

Assumption A: $K_{0}$ has an entire discrete spectrum such that

$$
\operatorname{spec}\left(K_{0}\right) \subseteq \mathbb{N}+\lambda
$$

for some $\lambda \geq 0$. 
Remark 2.2. Assumption A guarantees that $e^{\mathrm{i} 2 \pi K_{0}}=e^{\mathrm{i} 2 \pi \lambda}$. In particular, for any operator $V$, the map $t \mapsto e^{\mathrm{i} t K_{0}} V e^{-\mathrm{i} t K_{0}}$ is $2 \pi$-periodic.

The next assumption regards the existence of a bounded, time independent operator with unbounded orbits:

Assumption B: There exists an operator $A \in \mathcal{L}\left(\mathcal{H}^{r}\right)$, an initial datum $\psi_{0} \in \mathcal{H}^{r}$ and a real $\mu>0$ such that the Schrödinger equation

$$
\mathrm{i} \dot{\psi}=A \psi, \quad \psi(0)=\psi_{0}
$$

has a solution $\psi(t) \in \mathcal{H}^{r}$ fulfilling

$$
\|\psi(t)\|_{r} \geq C_{r} t^{\mu r}, \quad t \gg 1
$$

Despite Assumption B might seem very strong, in applications it can be verified quite easily. In Lemma 2.6 below we give some sufficient conditions on the operator $A$ to obtain (11).

The last assumption concerns smoothness in time of the map $t \mapsto \mathrm{e}^{-\mathrm{i} t K_{0}} A \mathrm{e}^{\mathrm{i} t K_{0}}$ :

Assumption C: The map $t \mapsto \mathrm{e}^{-\mathrm{i} t K_{0}} A \mathrm{e}^{\mathrm{i} t K_{0}} \in C^{\infty}\left(\mathbb{R} ; \mathcal{L}\left(\mathcal{H}^{r}\right)\right)$.

Here $C^{\infty}\left(\mathbb{R} ; \mathcal{L}\left(\mathcal{H}^{r}\right)\right)$ is the class of smooth maps from $\mathbb{R}$ to $\mathcal{L}\left(\mathcal{H}^{r}\right)$.

Remark 2.3. By Remark 2.2, $t \mapsto \mathrm{e}^{-\mathrm{i} t K_{0}} A \mathrm{e}^{\mathrm{i} t K_{0}} \in C^{\infty}\left(\mathbb{T} ; \mathcal{L}\left(\mathcal{H}^{r}\right)\right)$, namely the map and all its derivatives are $2 \pi$-periodic.

In applications, Assumption $\mathrm{C}$ can be verified by requiring $A$ and $K_{0}$ to be pseudodifferential operators and $K_{0}$ to fulfill an Egorov-like theorem.

Remark 2.4. We do not require explicitly $K_{0}$ to have constant spectral gaps; however, in applications, the only operators that we could find that verify both Assumption A and C have constant spectral gaps.

Our main result is the following one:

Theorem 2.5. Assume A, B, C. There exists $V_{A}(t) \in C^{\infty}\left(\mathbb{T}, \mathcal{L}\left(\mathcal{H}^{r}\right)\right)$ s.t. $K_{0}+V_{A}(t)$ generates unbounded orbits. More precisely there exists a smooth solution $\psi(t)$ of (1), with $\psi(t) \in \mathcal{H}^{r} \quad \forall t$, fulfilling (11). 
Proof. The proof is trivial. Define $V_{A}(t)$ as in (4). By Assumption A and C, it belongs to $C^{\infty}\left(\mathbb{T}, \mathcal{L}\left(\mathcal{H}^{r}\right)\right)$. The change of coordinates $\psi=e^{-\mathrm{i} t K_{0}} \varphi$ conjugates $\mathrm{i} \dot{\psi}=\left(K_{0}+V_{A}(t)\right) \psi$ to (10) and preserves the norm $\|\cdot\|_{r}$ for any time $t$. Then Assumption B implies the claim.

We comment now on Assumption B. First remark that a necessary condition for (11) to be fulfilled is that $\left[K_{0}, A\right] \neq 0$. Then a sufficient condition is that only a finite number of iterated commutators of $A$ and $K_{0}$ are not zero. We define $\operatorname{ad}_{A}(B):=[A, B]$.

Lemma 2.6. Assume that for some $N \in \mathbb{N}$ one has

$$
\operatorname{ad}_{A}^{j}\left(K_{0}\right) \neq 0, \quad \forall 1 \leq j \leq N, \quad \operatorname{ad}_{A}^{N+1}\left(K_{0}\right)=0
$$

Fix $r \in \mathbb{N}$ and choose $\psi_{0} \in \mathcal{H}^{r}$ such that

$$
\left[\operatorname{ad}_{A}^{N}\left(K_{0}\right)\right]^{r} \psi_{0} \neq 0
$$

Then there exists $C\left(r, N, \psi_{0}\right)>0$ such that the solution $\psi(t)$ of (11) with initial datum $\psi_{0}$ fulfills

$$
\|\psi(t)\|_{r} \geq C\left(r, N, \psi_{0}\right)\langle t\rangle^{r N}, \quad t \gg 1
$$

The proof of the Lemma is postponed in Appendix $\mathrm{A}$.

Remark 2.7. Condition (12) can be replaced by $\operatorname{ad}_{A}^{N}\left(K_{0}^{\prime}\right) \neq 0, \operatorname{ad}_{A}^{N+1}\left(K_{0}^{\prime}\right)=0$ where $K_{0}^{\prime}$ is any operator defining norms equivalent to $\|\cdot\|_{r}$.

We conclude this section with an example of an operator $A$ with absolutely continuous spectrum which has unbounded orbits; such example will guide us in the applications:

Example 2.8. Let $H^{r}(\mathbb{T})=\operatorname{Dom}\left(\left(1-\partial_{x x}\right)^{r / 2}\right)$ be the classical Sobolev space on the one dimensional torus $\mathbb{T}$. Define $A=v(x)$ (multiplication operator), with $v(x) \in C^{\infty}(\mathbb{T}, \mathbb{R})$ and $\nabla v \not \equiv 0$. Then the equation

$$
\mathrm{i} \dot{\varphi}=v(x) \varphi, \quad \text { with } \varphi(0) \in H^{r}(\mathbb{T}) \quad \text { and } \quad(\nabla v) \cdot \varphi(0) \not \equiv 0
$$

has a solution $\varphi(t) \in H^{r}(\mathbb{T})$ fulfilling (11) with $\mu=1$. This follows applying Lemma 2.6 and Remark 2.7 with $K_{0}^{\prime}=\partial_{x}$ and noting that $\left[v(x), \partial_{x}\right] \neq 0,\left[v(x),\left[v(x), \partial_{x}\right]\right]=0$. 


\section{Applications}

In this section we apply Theorem 2.5 to three different time dependent linear Schrödinger equations. In each case we construct a periodic in time perturbation which induces growth of norms.

In this section, for $\Omega \subset \mathbb{R}^{d}$ and $\mathcal{F}$ a Fréchet space, we will denote by $C_{b}^{m}(\Omega, \mathcal{F})$ the space of $C^{m}$ maps $f: \Omega \ni x \mapsto f(x) \in \mathcal{F}$, such that, for every seminorm $\|\cdot\|_{j}$ of $\mathcal{F}$ one has

$$
\sup _{x \in \Omega}\left\|\partial_{x}^{\alpha} f(x)\right\|_{j}<+\infty, \quad \forall \alpha \in \mathbb{N}^{d}:|\alpha| \leq m .
$$

If (15) is true $\forall m$, we say $f \in C_{b}^{\infty}(\Omega, \mathcal{F})$.

\subsection{Harmonic oscillator on $\mathbb{R}$}

Consider the Schrödinger equation

$$
\mathrm{i} \dot{\psi}=\frac{1}{2}\left(-\partial_{x}^{2}+x^{2}\right) \psi+V\left(t, x, D_{x}\right) \psi, \quad x \in \mathbb{R} .
$$

Here $K_{0}:=\frac{1}{2}\left(-\partial_{x}^{2}+x^{2}\right)$ is the Harmonic oscillator, the scale of Hilbert spaces is defined as usual by $\mathcal{H}^{r}=\operatorname{Dom}\left(K_{0}^{r}\right)$, and the base space $\left(\mathcal{H}^{0},\langle\cdot, \cdot\rangle,\right)$ is $L^{2}(\mathbb{R}, \mathbb{C})$ with its standard scalar product.

The perturbation $V$ is chosen as the Weyl quantization of a symbol belonging to the following class

Definition 3.1. A function $f$ is a symbol of order $\rho \in \mathbb{R}$ if $f \in C^{\infty}\left(\mathbb{R}_{x} \times \mathbb{R}_{\xi}, \mathbb{R}\right)$ and $\forall \alpha, \beta \in \mathbb{N}$, there exists $C_{\alpha, \beta}>0$ such that

$$
\left|\partial_{x}^{\alpha} \partial_{\xi}^{\beta} f(x, \xi)\right| \leq C_{\alpha, \beta}\left(1+|x|^{2}+|\xi|^{2}\right)^{\rho-\frac{|\beta|+|\alpha|}{2}} .
$$

We will write $f \in S_{\mathrm{ho}}^{\rho}$.

We endow $S_{\text {ho }}^{\rho}$ with the family of seminorms

$$
\wp_{j}^{\rho}(f):=\sum_{|\alpha|+|\beta| \leq j} \sup _{(x, \xi) \in \mathbb{R}^{2}} \frac{\left|\partial_{x}^{\alpha} \partial_{\xi}^{\beta} f(x, \xi)\right|}{\left[1+|x|^{2}+|\xi|^{2}\right]^{\rho-\frac{\beta+\alpha}{2}}}, \quad j \in \mathbb{N} \cup\{0\} .
$$

Such seminorms turn $S_{\text {ho }}^{\rho}$ into a Fréchet space.

If a symbol $f$ depends on additional parameters (e.g. it is time dependent), we ask that 
all the seminorms are uniform w.r.t. such parameters.

To a symbol $f \in S_{\text {ho }}^{\rho}$ we associate the operator $f\left(x, D_{x}\right)$ by standard Weyl quantization

$$
\left(f\left(x, D_{x}\right) \psi\right)(x):=\frac{1}{2 \pi} \iint_{y, \xi \in \mathbb{R}} \mathrm{e}^{\mathrm{i}(x-y) \xi} f\left(\frac{x+y}{2}, \xi\right) \psi(y) \mathrm{d} y \mathrm{~d} \xi .
$$

Definition 3.2. We say that $F \in \mathcal{A}_{\rho}$ if it is a pseudodifferential operator with symbol of class $S_{\mathrm{ho}}^{\rho}$, i.e., if there exists $f \in S_{\mathrm{ho}}^{\rho}$ such that $F=f\left(x, D_{x}\right)$.

Remark 3.3. The harmonic oscillator $K_{0}$ has symbol given by $x^{2}+\xi^{2}$; by our definition $K_{0} \in \mathcal{A}_{1}$.

As usual we give $\mathcal{A}_{\rho}$ a Fréchet structure by endowing it with the seminorms of the symbols.

Our first application is to construct a time dependent pseudodifferential operator of order 0 which provokes growth of Sobolev norms. In such a way we obtain an alternative, simpler construction of the result of Delort [Del14].

Theorem 3.4. There exists a time periodic pseudodifferential operator of order $0, V \in$ $C_{b}^{\infty}\left(\mathbb{T}, \mathcal{A}_{0}\right)$, and for any $r \in \mathbb{N}$ a constant $C_{r}>0$ and a smooth solution $\psi(t)$ of (16) s.t. for any $t \geq 0, \psi(t) \in \mathcal{H}^{r}$ and satisfies $\|\psi(t)\|_{r} \geq C_{r} t^{r}$ for $t$ large enough.

Proof. We verify that Assumptions A, B, and C are met.

Assumption A: It follows from $\sigma\left(K_{0}\right)=\left\{n+\frac{1}{2}\right\}_{n \geq 0}$.

Assumption B: We define $A$ by its action on the Hermite functions $\left(\mathbf{e}_{n}\right)_{n \in \mathbb{N}_{0}}$ (which are eigenvectors of the Harmonic oscillator and form a basis of $\left.L^{2}(\mathbb{R})\right)$. We take $\delta \neq 0$ arbitrary and define

$$
A \mathbf{e}_{0}:=\delta \mathbf{e}_{1}, \quad A \mathbf{e}_{n}:=\delta\left(\mathbf{e}_{n+1}+\mathbf{e}_{n-1}\right) \quad \text { for } n \geq 1
$$

the action of $A$ is extended on all $\mathcal{H}^{r}$ by linearity, giving $A \psi=\delta \sum_{n \geq 0}\left(\psi_{n-1}+\psi_{n+1}\right) \mathbf{e}_{n}$, where we defined $\psi_{n}=\left\langle\psi, \mathbf{e}_{n}\right\rangle$ for $n \geq 0$ and $\psi_{-1}=0$. Clearly $A \in \mathcal{L}\left(\mathcal{H}^{r}\right) \forall r \geq 0$.

By Lemma 3.10 below its propagator $e^{-\mathrm{i} t A}$ has unbounded orbits fulfilling (11) with $\mu=1$ and any initial datum.

Assumption C: By Lemma 3.13 below, $A$ is a pseudodifferential operator of order 0, $A \in \mathcal{A}_{0}$. By Egorov theorem for the Harmonic oscillator HR82b, HR82a (and using the periodicity of the flow of $\left.K_{0}\right) t \mapsto e^{-\mathrm{i} t K_{0}} A e^{\mathrm{i} t K_{0}} \equiv V_{A}(t) \in C_{b}^{\infty}\left(\mathbb{T}, \mathcal{A}_{0}\right)$. This can be seen e.g. by remarking that the symbol of $V_{A}(t)$ is $a \circ \phi_{h o}^{t}$, where $a \in S_{\text {ho }}^{0}$ is the symbol 
of $A$ and $\phi_{h o}^{t}$ is the time $t$ flow of the harmonic oscillator; explicitly

$$
\left(a \circ \phi_{h o}^{t}\right)(x, \xi)=a(x \cos t+\xi \sin t,-x \sin t+\xi \cos t) .
$$

Remark 3.5. The parameter $\delta$ in (17) can be arbitrary small; therefore, also the perturbation $V_{A}(t)$ can be arbitrary small in size.

Remark 3.6. Consider (16) with the perturbation $V_{A}\left(\omega t, x, D_{x}\right), \omega \in \mathbb{R}$, which is periodic in time with frequency $\omega$. Then it is proved in [Bam18] that for any $\delta$ sufficiently small, there exists a Cantor set $\mathcal{O}_{\delta} \subset[0,1]$ such that if $\omega \in \mathcal{O}_{\delta}$, each solution of (16) fulfills (8). It is clear therefore that the growth of Sobolev norms depends on resonance properties of the frequency $\omega$.

Remark 3.7. In the basis of Hermite functions, the operator $A$ is the discrete Laplacian on the half line $\ell^{2}\left(\mathbb{N}_{0}\right)$ with Dirichlet boundary conditions, hence it has absolutely continuous spectrum.

Remark 3.8. The Fourier transform $\left(\psi_{n}\right)_{n \in \mathbb{N}_{0}} \mapsto \sum_{n \geq 0} \psi_{n} \sin ((n+1) y)$ maps $\mathcal{H}^{r}$ in $H^{r}(\mathbb{T})$ and conjugates $A$ to the multiplication operator by $\delta \cos (x)$; therefore we are in the framework of Example 2.8.

Remark 3.9. One has $V_{A} \in C^{\infty}\left(\mathbb{T}, \mathcal{L}\left(\mathcal{H}^{r}\right)\right)$ for any $r \geq 0$. Indeed by CalderonVaillancourt theorem for the class $\mathcal{A}_{\rho}$ (see e.g. Rob877), for any $r \in \mathbb{N}$, there exists $N \in \mathbb{N}$ and $C_{r, N}>0$ such that

$$
\sup _{t \in \mathbb{T}}\left\|\partial_{t}^{\ell} V_{A}(t)\right\|_{\mathcal{L}\left(\mathcal{H}^{r}\right)} \leq C_{r, N} \wp_{N}^{\rho}\left(\partial_{t}^{\ell} a(t, x, \xi)\right)<\infty, \quad \forall \ell \in \mathbb{N}_{0}
$$

where $a(t, x, \xi) \equiv\left(a \circ \phi_{h o}^{t}\right)(x, \xi)$ is the symbol of $V_{A}(t)$, see $(18)$, and belongs to $C_{b}^{\infty}\left(\mathbb{R}, S_{\mathrm{ho}}^{0}\right)$.

First we prove that the Hamiltonian $A$ generates unbounded orbits:

Lemma 3.10. Let $A$ be defined in (17) and consider the equation $\mathrm{i} \dot{\psi}=A \psi$. For any $r \in \mathbb{N}$, any nonzero $\psi_{0} \in \mathcal{H}^{r}$, there exists a constant $C_{r, \psi_{0}}>0$ such that $\left\|e^{-\mathrm{i} t A} \psi_{0}\right\|_{r} \geq$ $C_{r, \psi_{0}}\langle t\rangle^{r}$ for $t \gg 1$.

Proof. Remark 3.8 gives essentially a proof of the statement. Alternatively, one can also apply Lemma 2.6 as follows. Let $\mathbf{e}_{n}$ be the $n^{\text {th }}$ Hermit function; then a direct computation using (17) and $K_{0} \mathbf{e}_{n}=\left(n+\frac{1}{2}\right) \mathbf{e}_{n}$ shows that

$$
\left[A, K_{0}\right] \mathbf{e}_{n}=\delta\left(\mathbf{e}_{n-1}-\mathbf{e}_{n+1}\right), \quad\left[A,\left[A, K_{0}\right]\right] \mathbf{e}_{n}=0, \quad \forall n \in \mathbb{N}_{0}
$$


Then we apply Lemma 2.6 with $N=1$ ( (13) is fulfilled for any nontrivial $\psi_{0} \in \mathcal{H}^{r}$ ).

Finally we show that $A$ is a pseudodifferential operator; to this aim, it is necessary to read the pseudodifferential properties of an operator by its matrix coefficients on the basis of Hermit functions. Any linear self-adjoint operator $A: \mathcal{H}^{\infty} \rightarrow \mathcal{H}^{-\infty}$ is completely determined by its matrix

$$
M^{(A)}: \mathbb{N}_{0} \times \mathbb{N}_{0} \rightarrow \mathbb{R}, \quad(m, n) \rightarrow\left\langle A \mathbf{e}_{m}, \mathbf{e}_{n}\right\rangle
$$

Define the discrete difference operator $\triangle$ on a function $M: \mathbb{N}_{0} \times \mathbb{N}_{0} \rightarrow \mathbb{R}$ by

$$
(\triangle M)(m, n):=M(m+1, n+1)-M(m, n),
$$

and its powers $\Delta^{\gamma}$ by $\triangle$ applied $\gamma$-times. Now we have the following

Definition 3.11. A symmetric function $M: \mathbb{N}_{0} \times \mathbb{N}_{0} \rightarrow \mathbb{R}$ will be said to be a symbol matrix of order $\rho$ if for any $\gamma, N \in \mathbb{N}$, there exists $C_{\gamma, N}>0$ such that

$$
\left|\left(\triangle^{\gamma} M\right)(m, n)\right| \leq C_{\gamma, N} \frac{(1+m+n)^{\rho-|\gamma|}}{(1+|m-n|)^{N}} .
$$

The connection between pseudodifferential operators of order $\rho$ and symbol matrices of order $\rho$ is given by Chodosh's characterization [Cho11]:

Theorem 3.12 (Chodosh's characterization). A selfadjoint operator A belongs to $\mathcal{A}_{\rho}$ if and only if its matrix $M^{(A)}$ (as defined in (19)) is a symbol matrix of order $\rho$.

We can now prove:

Lemma 3.13. The operator $A$ defined in (17) belongs to $\mathcal{A}_{0}$.

Proof. By formula (17), the matrix of $A$ is given by

$$
M^{(A)}(m, n):=\left\langle A \mathbf{e}_{m}, \mathbf{e}_{n}\right\rangle=\delta_{n+1, m}+\delta_{n-1, m}, \quad n, m \in \mathbb{N}_{0}
$$

It is a trivial computation to verify that $M^{(A)}$ is a symbol matrix of order 0 , hence by Theorem 3.12 it is a pseudodifferential operator in $\mathcal{A}_{0}$.

\section{$3.2 \quad$ Half-wave equation on $\mathbb{T}$}

The half-wave equation on $\mathbb{T}$ is given by

$$
\mathrm{i} \dot{\psi}=\left|D_{x}\right| \psi+V\left(t, x, D_{x}\right) \psi, \quad x \in \mathbb{T}
$$


Here $\left|D_{x}\right| \psi$ is the Fourier multiplier defined by

$$
\left|D_{x}\right| \psi:=\sum_{j \in \mathbb{Z}}|j| \psi_{j} e^{\mathrm{i} j x},
$$

where $\psi_{j}:=\int_{\mathbb{T}} \psi(x) e^{-\mathrm{i} j x} \mathrm{~d} x$ is the $j^{\text {th }}$ Fourier coefficient.

We recall now the usual class of pseudodifferential operators on $\mathbb{T}$. For a function $f: \mathbb{T} \times \mathbb{Z} \rightarrow \mathbb{R}$, define the difference operator $\triangle f(x, j):=f(x, j+1)-f(x, j)$. Then we have

Definition 3.14. A function $f: \mathbb{T} \times \mathbb{Z} \rightarrow \mathbb{R}$, will be called a symbol of order $\rho \in \mathbb{R}$ if $x \mapsto f(x, j)$ is smooth for any $j \in \mathbb{Z}$ and $\forall \alpha, \beta \in \mathbb{N}$, there exists $C_{\alpha, \beta}>0$ s.t.

$$
\left|\partial_{x}^{\alpha} \triangle^{\beta} f(x, j)\right| \leq C_{\alpha, \beta}\langle j\rangle^{\rho-\beta}
$$

We will write $f \in S_{\text {to }}^{\rho}$.

Again we endow $S_{\text {to }}^{\rho}$ with the family of seminorms

$$
\wp_{j}^{\rho}(f):=\sum_{\alpha+\beta \leq j} \sup _{(x, j) \in \mathbb{T} \times \mathbb{Z}}\langle j\rangle^{-\rho+\beta}\left|\partial_{x}^{\alpha} \triangle_{\xi}^{\beta} f(x, j)\right|, \quad j \in \mathbb{N}_{0}
$$

If a symbol $f$ depends on additional parameters (e.g. it is time dependent), we ask that the constants $C_{\alpha, \beta}$ are uniform w.r.t. such parameters.

To a symbol $f \in S_{\text {to }}^{\rho}$ we associate the operator $f\left(x, D_{x}\right)$ by standard quantization:

$$
\left(f\left(x, D_{x}\right) \psi\right)(x):=\sum_{j \in \mathbb{Z}} f(x, j) \psi_{j} e^{\mathrm{i} j x} .
$$

Then we have the following

Definition 3.15. We say that $F \in \mathcal{A}_{\rho}$ if it is a pseudodifferential operator with symbol of class $S_{\mathrm{to}}^{\rho}$, i.e., if there exist $f \in S_{\mathrm{to}}^{\rho}$ such that $F=f\left(x, D_{x}\right)$.

Remark 3.16. The operator $\left|D_{x}\right|$ has symbol given by $|j|$; therefore $\left|D_{x}\right| \in \mathcal{A}_{1}$.

As usual we give $\mathcal{A}_{\rho}$ a Fréchet structure by endowing it with the seminorms of the symbols.

In this case we have:

Theorem 3.17. Consider the half-wave equation (20). There exist a pseudodifferential operator of order $0, V \in C^{\infty}\left(\mathbb{T}, \mathcal{A}_{0}\right)$, and for any $r \in \mathbb{N}$ a constant $C_{r}>0$ and a solution 
$\psi(t)$ of (201) such that, for any $t \geq 0, \psi(t) \in H^{r}(\mathbb{T})$ and satisfies $\|\psi(t)\|_{H^{r}(\mathbb{T})} \geq C_{r} t^{r}$ for $t$ large enough.

Proof. First we show how to put ourselves in the setting of the abstract problem. Define

$$
K_{0}:=\left|D_{x}\right|+\lambda, \quad \lambda>0
$$

The space $\mathcal{H}^{r}:=\operatorname{Dom}\left(K_{0}^{r}\right)$ coincides with $H^{r}(\mathbb{T})$, with equivalent norms. We take perturbations of the form $V\left(t, x, D_{x}\right)=\lambda\left(1+e^{-\mathrm{i} t K_{0}} A e^{\mathrm{i} t K_{0}}\right)$, so that (20) becomes

$$
\mathrm{i} \dot{\psi}=K_{0} \psi+V_{A}\left(t, x, D_{x}\right) \psi, \quad V_{A}\left(t, x, D_{x}\right):=\lambda e^{-\mathrm{i} t K_{0}} A e^{\mathrm{i} t K_{0}},
$$

and we are back to the abstract setting. We need only to verity Assumptions A, B, C.

Assumption A: Trivial, since $\sigma\left(K_{0}\right)=\{j+\lambda\}_{j \in \mathbb{N}_{0}}$.

Assumption B: Take $A$ and the initial datum $\psi_{0}$ as in Example 2.8.

Assumption C: One has $A \in \mathcal{A}_{0}$ and $K_{0} \in \mathcal{A}_{1}$. By a classical result of Hörmander [Hör85] (see also [DG75]), $t \mapsto e^{-\mathrm{i} t K_{0}} A e^{\mathrm{it} K_{0}} \in C^{\infty}\left(\mathbb{R}, \mathcal{A}_{0}\right)$; actually, being periodic in time, it belongs to $C^{\infty}\left(\mathbb{T}, \mathcal{A}_{0}\right)$.

Remark 3.18. The parameter $\lambda$ can be arbitrary small; therefore also in this case $V\left(t, x, D_{x}\right)$ can be arbitrary small in size.

Remark 3.19. By Calderon-Vaillancourt theorem, $V\left(t, x, D_{x}\right) \in C^{\infty}\left(\mathbb{T}, \mathcal{L}\left(\mathcal{H}^{r}\right)\right) \forall r \in \mathbb{N}$.

\subsection{Schrödinger-Dirac equation on Zoll manifolds}

Consider the Schrödinger-Dirac equation on a Zoll manifold $M$ (e.g., $M$ can be a $n$ dimensional sphere)

$$
\mathrm{i} \dot{\psi}=\sqrt{-\Delta_{g}+m^{2}} \psi+V\left(t, x, D_{x}\right) \psi, \quad x \in M
$$

here $m \neq 0$ is a real number and $-\Delta_{g}$ is the positive Laplace-Beltrami operator on $M$. Let $H^{r}(M)=\operatorname{Dom}\left(\left(1-\Delta_{g}\right)^{r / 2}\right), r \geq 0$, the usual scale of Sobolev spaces on $M$. Finally we denote by $S_{\mathrm{cl}}^{\rho}$ the space of classical real valued symbols of order $\rho \in \mathbb{R}$ on the cotangent $T^{*}(M)$ of $M$ (see Hörmander [Hör85] for more details).

Definition 3.20. We say that $F \in \mathcal{A}_{\rho}$ if it is a pseudodifferential operator (in the sense of Hörmander [Hör85]) with symbol of class $S_{\mathrm{cl}}^{\rho}$.

Remark 3.21. The operator $\sqrt{-\Delta_{g}+m^{2}}$ belongs to $\mathcal{A}_{1}$ [Hör85]. 
Remark 3.22. By $C d V 79$, there exist $c_{0}, c_{1}>0$ such that

$$
\sigma\left(\sqrt{-\Delta_{g}+m^{2}}\right) \subseteq \bigcup_{j \geq 0}\left[j+c_{0}-\frac{c_{1}}{j}, j+c_{0}+\frac{c_{1}}{j}\right],
$$

so in this case the spectral gaps are only asymptotically constant.

We have the following

Theorem 3.23. Consider the Schrödinger-Dirac equation (21). There exists a pseudodifferential operator of order $0, V \in C^{\infty}\left(\mathbb{T}, \mathcal{A}_{0}\right)$, and for any $r \in \mathbb{N}$ a constant $C_{r}>0$ and a solution $\psi(t)$ of (21) fulfilling $\psi(t) \in H^{r}(M)$ for any time $t$, and

$$
\|\psi(t)\|_{H^{r}(M)} \geq C_{r} t^{r}, \quad t \gg 1
$$

Proof. To begin with we show how to put ourselves in the abstract setup. So first we define the operator $K_{0}$. This is achieved by exploiting the spectral properties of the operator $-\Delta_{g}$. Applying Theorem 1 of Colin de Verdière [CdV79, there exists a pseudodifferential operator $Q$ of order -1 , commuting with $-\Delta_{g}$, such that $\operatorname{Spec}\left[\sqrt{-\Delta_{g}+m^{2}}+Q\right] \subseteq \mathbb{N}+\lambda$ with some $\lambda>0$. So we define

$$
K_{0}:=\sqrt{-\Delta_{g}+m^{2}}+Q \in \mathcal{A}_{1}
$$

Since $Q \in \mathcal{A}_{-1}$, the space $\mathcal{H}^{r}:=\operatorname{Dom}\left(K_{0}^{r}\right), r \geq 0$, coincides with the classical Sobolev space $H^{r}(M)$ and one has the equivalence of norms

$$
c_{r}\|\psi\|_{H^{r}(M)} \leq\|\psi\|_{r} \leq \widetilde{c}_{r}\|\psi\|_{H^{r}(M)}, \quad \forall r \in \mathbb{R} .
$$

We take the perturbation of the form $V\left(t, x, D_{x}\right)=Q+e^{-\mathrm{i} t K_{0}} A e^{\mathrm{i} t K_{0}}$, so that (21) becomes

$$
\mathrm{i} \dot{\psi}=K_{0} \psi+V_{A}\left(t, x, D_{x}\right), \quad V_{A}\left(t, x, D_{x}\right)=e^{-\mathrm{i} t K_{0}} A e^{\mathrm{i} t K_{0}}
$$

and we are back to the abstract setting. We need only to verify Assumptions A, B, C.

Assumption A: True by construction.

Assumption B: It follows by a trivial variant of Example 2.8, Choose any nonconstant $v(x) \in C^{\infty}(M, \mathbb{R})$, define $A$ as the multiplication operator by $v(x)$, and take an initial datum $\psi_{0} \in \mathcal{H}^{r}$ fulfilling $\left(\nabla_{g} v(x)\right) \psi_{0} \not \equiv 0$. For example, $v(x)$ can be any noncostant eigenfunction of $-\Delta_{g}$. Then the Schrödinger equation (10) has orbits fulfilling (11) with $\mu=1$ (it is enough to apply Lemma 2.6 and Remark 2.7 using $\left[\nabla_{g}, v(x)\right] \neq 0$, 
$\left.\left[\left[\nabla_{g}, v(x)\right], v(x)\right]=0\right)$.

Assumption C: One has $A \in \mathcal{A}_{0}$ and $K_{0} \in \mathcal{A}_{1}$. Then $e^{-\mathrm{i} t K_{0}} A e^{\mathrm{i} t K_{0}} \in C^{\infty}\left(\mathbb{R}, \mathcal{A}_{0}\right)$ by a classical result of Hörmander Hör85.

Remark 3.24. In this case, the perturbation $V\left(t, x, D_{x}\right)$ cannot be chosen arbitrary small in size, since we have to add the smoothing operator $Q$ to correct the spectral gaps.

Remark 3.25. One could also choose $V\left(t, x, D_{x}\right)$ as $e^{-\mathrm{i} t \sqrt{-\Delta_{g}+m^{2}}} \epsilon A e^{\mathrm{i} t \sqrt{-\Delta_{g}+m^{2}}}$; in such a way the perturbation is arbitrary small in size and fulfills Assumption $\mathrm{C}$ (again by [Hör85]), but it is not periodic in time.

\section{A Proof of Lemma 2.6}

Since the linear operator $\operatorname{ad}_{A}$ fulfills Leibniz rule, for any $M, r \in \mathbb{N}$ one has the identity

$$
\operatorname{ad}_{A}^{M}\left(K_{0}^{2 r}\right)=\sum_{k_{1}+\ldots+k_{2 r}=M}\left(\begin{array}{c}
M \\
k_{1} \cdots k_{2 r}
\end{array}\right) \operatorname{ad}_{A}^{k_{1}}\left(K_{0}\right) \operatorname{ad}_{A}^{k_{2}}\left(K_{0}\right) \cdots \operatorname{ad}_{A}^{k_{2 r}}\left(K_{0}\right)
$$

If $M \geq 2 r N+1$ then in (23) at least one index $k_{j}$ is greater equal $N+1$, so by assumption (12) the whole expression is zero. By the same argument, if $M=2 r N$ then the only term not null in (23) is the one with $k_{j}=N \forall j$, which is $\left[\operatorname{ad}_{A}^{N}\left(K_{0}\right)\right]^{2 r}$.

Consider now the solution $\psi(t) \equiv e^{-i t A} \psi_{0}$ of equation (10). Since $A$ is self-adjoint,

$$
\|\psi(t)\|_{r}^{2} \equiv\left\langle e^{\mathrm{i} t A} K_{0}^{2 r} e^{-\mathrm{i} t A} \psi_{0}, \psi_{0}\right\rangle
$$

where we used $\|\psi\|_{r}^{2} \equiv\left\langle K_{0}^{2 r} \psi, \psi\right\rangle$. Now we use the Lie formula $e^{\mathrm{i} t A} B e^{-\mathrm{i} t A} \equiv \sum_{j \geq 0} \frac{(\mathrm{i} t)^{j}}{j !} \operatorname{ad}_{A}^{j}(B)$, assumption (12) and our previous considerations to obtain

$$
e^{\mathrm{i} t A} K_{0}^{2 r} e^{-\mathrm{i} t A}=\sum_{M=0}^{2 r N} \frac{(\mathrm{i} t)^{M}}{M !} \operatorname{ad}_{A}^{M}\left(K_{0}^{2 r}\right) \equiv \frac{(\mathrm{i} t)^{2 r N}}{(2 r N) !}\left[\operatorname{ad}_{A}^{N}\left(K_{0}\right)\right]^{2 r}+O\left(t^{2 r N-1}\right)
$$

provided $\left[\operatorname{ad}_{A}^{N}\left(K_{0}\right)\right]^{r} \psi_{0} \neq 0$, it follows that

$$
\liminf _{t \rightarrow+\infty} \frac{\|\psi(t)\|_{r}^{2}}{\left|t^{2 r N}\right|} \geq \frac{1}{(2 r N) !}\left|\left\langle\left[\operatorname{ad}_{A}^{N}\left(K_{0}\right)\right]^{2 r} \psi_{0}, \psi_{0}\right\rangle\right|>0
$$

In particular there exists a constant $C\left(r, N, \psi_{0}\right)>0$ such that (14) holds true. 


\section{References}

[Bam18] D. Bambusi. Reducibility of 1-d Schrödinger equation with time quasiperiodic unbounded perturbations, I. Trans. Amer. Math. Soc., 370(3):1823$1865,2018$.

[BGMR17] D. Bambusi, B. Grébert, A. Maspero, and D. Robert. Growth of Sobolev norms for abstract linear Schrödinger Equations. ArXiv e-prints, arXiv:1706.09708, 2017.

[BGMR18] D. Bambusi, B. Grébert, A. Maspero, and D. Robert. Reducibility of the quantum harmonic oscillator in $d$-dimensions with polynomial timedependent perturbation. Anal. PDE, 11(3):775-799, 2018.

[Bou99] J. Bourgain. Growth of Sobolev norms in linear Schrödinger equations with quasi-periodic potential. Comm. Math. Phys., 204(1):207-247, 1999.

[CdV79] Y. Colin de Verdière. Sur le spectre des opérateurs elliptiques à bicaractéristiques toutes périodiques. Comment. Math. Helv., 54(3):508-522, 1979.

[Cho11] O. Chodosh. Infinite matrix representations of isotropic pseudodifferential operators. Methods Appl. Anal., 18(4):351-371, 2011.

[CKSTT] J. Colliander, M. Keel, G. Staffilani, H. Takaoka, and T. Tao. Transfer of energy to high frequencies in the cubic defocusing nonlinear Schrödinger equation. Invent. Math., 181(1):39-113, 2010.

[Com87] M. Combescure. The quantum stability problem for time-periodic perturbations of the harmonic oscillator. Ann. Inst. H. Poincaré Phys. Théor., 47(1):63-83, 1987.

[Com93] J.-M. Combes. Connections between quantum dynamics and spectral properties of time-evolution operators. In Differential Equations with Applications to Mathematical Physics, volume 192, pages 59-68. Elsevier, 1993.

[CY00] L. Chierchia and J. You. KAM tori for 1D nonlinear wave equations with periodic boundary conditions. Comm. Math. Phys., 211(2):497-525, 2000.

[Del14] J.-M. Delort. Growth of Sobolev norms for solutions of time dependent Schrödinger operators with harmonic oscillator potential. Comm. Partial Differential Equations, 39(1):1-33, 2014. 
[DG75] J. Duistermaat and V. Guillemin. The spectrum of positive elliptic operators and periodic bicharacteristics. Invent. Math., 29(1):39-79, 1975.

[FHW14] D. Fang, Z. Han, and W.-M. Wang. Bounded Sobolev norms for KleinGordon equations under non-resonant perturbation. J. Math. Phys., 55(12):121503, 11, 2014.

[GG17] P. Gérard and S. Grellier. The cubic Szegő equation and Hankel operators. Astérisque, (389):vi+112, 2017.

[GHP16] M. Guardia, E. Haus, and M. Procesi. Growth of Sobolev norms for the analytic NLS on $\mathbb{T}^{2}$. Adv. Math., 301:615-692, 2016.

[GP16a] B. Grébert and E. Paturel. KAM for the Klein Gordon equation on $\mathbb{S}^{d}$. ArXiv e-prints, arXiv:1601.00610, 2016.

[GP16b] B. Grébert and E. Paturel. On reducibility of quantum Harmonic Oscillator on $\mathbb{R}^{d}$ with quasiperiodic in time potential. ArXiv e-prints, arXiv:1603.07455, 2016.

[GT11] B. Grébert and L. Thomann. KAM for the quantum harmonic oscillator. Comm. Math. Phys., 307(2):383-427, 2011.

[Gua89] I. Guarneri. Spectral properties of quantum diffusion on discrete lattices. Europhysics Letters, 10(2):95, 1989.

[Gua14] M. Guardia. Growth of Sobolev norms in the cubic nonlinear Schrödinger equation with a convolution potential. Comm. Math. Phys., 329(1):405-434, 2014.

[GY00] S. Graffi and K. Yajima. Absolute continuity of the Floquet spectrum for a nonlinearly forced harmonic oscillator. Comm. Math. Phys., 215(2):245-250, 2000 .

[Han14] Z. Hani. Long-time instability and unbounded Sobolev orbits for some periodic nonlinear Schrödinger equations. Arch. Ration. Mech. Anal., 211(3):929-964, 2014.

[Hör85] L. Hörmander. The analysis of linear partial differential operators IV. Grundlehren der mathematischen Wissenschaften 256. Springer-Verlag, 1985. 
[HP15] E. Haus and M. Procesi. Growth of Sobolev norms for the quintic NLS on $\mathbb{T}^{2}$. Anal. PDE, 8(4):883-922, 2015.

[HPTV15] Z. Hani, B. Pausader, N. Tzvetkov, and N. Visciglia. Modified scattering for the cubic Schrödinger equation on product spaces and applications. Forum Math. Pi, 3:e4, 63, 2015.

[HR82a] B. Helffer and D. Robert. Asymptotique des niveaux d'énergie pour des hamiltoniens à un degré de liberté. Duke Math. J., 49(4):853-868, 1982.

[HR82b] B. Helffer and D. Robert. Propriétés asymptotiques du spectre d'opérateurs pseudodifférentiels sur $\mathbf{R}^{n}$. Comm. Partial Differential Equations, 7(7):795$882,1982$.

[Mon17] R. Montalto. A reducibility result for a class of linear wave equations on $\mathbb{T}^{d}$. IMRN, rnx167, DOI:10.1093/imrn/rnx167, 2017.

[MR17] A. Maspero and D. Robert. On time dependent Schrödinger equations: Global well-posedness and growth of Sobolev norms. Journal of Functional Analysis, 273(2):721 - 781, 2017.

[Rob87] D. Robert. Autour de l'approximation semi-classique. PM 68. Birkhäuser, 1987.

[Wan08] W.-M. Wang. Pure point spectrum of the Floquet Hamiltonian for the quantum harmonic oscillator under time quasi-periodic perturbations. Comm. Math. Phys., 277(2):459-496, 2008. 